\title{
COVARIANCE AND RELAXATION TIME IN FINITE MARKOV CHAINS
}

\author{
JULIAN KEILSON \\ University of Rochester \\ William E. Simon Graduate School of Business Administration \\ Rochester, NY 14627 USA
}

(Received February, 1998; Revised March, 1998)

\begin{abstract}
The relaxation time $T_{R E L}$ of a finite ergodic Markov chain in continuous time, i.e., the time to reach ergodicity from some initial state distribution, is loosely given in the literature in terms of the eigenvalues $\lambda_{j}$ of the infinitesimal generator $\underline{Q}$. One uses $T_{R E L}=\theta^{-1}$ where $\theta=\min _{\lambda_{j}} \neq 0$ $\left\{\operatorname{Re} \lambda_{j}[-Q]\right\}$. This paper establishes for the relaxation time $\theta^{-1}$ the theoretical solidity of the time reversible case. It does so by examining the structure of the quadratic distance $d(t)$ to ergodicity. It is shown that, for any function $f(j)$ defined for states $j$, the correlation function $\rho_{f}(\tau)$ has the bound $\left|\rho_{f}(\tau)\right| \leq \exp [-\theta|\tau|]$ and that this inequality is tight. The argument is almost entirely in the real domain.
\end{abstract}

Key words: Finite Markov Chains, Covariance, Relaxation Time.

AMS subject classifications: $60 \mathrm{~J} 27$.

\section{Introduction}

Let $J(t)$ be any ergodic Finite Markov Chain in continuous time with generator $\underline{\underline{Q}}$. A single underscore will be used to denote vectors and a double underscore will be

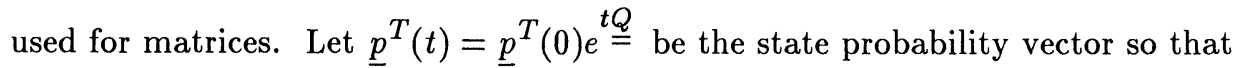

$$
\lim _{t \rightarrow \infty} \underline{p}^{T}(t)=\underline{e}^{T}=\left(e_{n}\right)_{1}^{K}>\underline{0}^{T} ; \underline{e}^{T} \underline{\underline{Q}}=\underline{0}^{T} .
$$

We are interested in the relaxation time of $J(t)$. For time-reversible chains where all eigenvalues of $\underline{\underline{Q}}$ are real the relaxation time is well understood (cf. Keilson [1]). For more general chains with real eigenvalues and eigenvalues occurring in complex conjugate pairs, all eigenvalues $\lambda_{j}[\underline{\underline{Q}}]$ other than zero have $\operatorname{Re} \lambda_{j}[\underline{\underline{Q}}]<0$ (see appendix). Let $\theta=\min _{\lambda_{j} \neq 0}\left\{\operatorname{Re} \lambda_{j}[-\underline{\underline{Q}}]\right\}$. The value $T_{R E L}=\theta^{-1}$ is employed loosely for the relaxation time in the literature. This paper establishes for the relaxation time 
$\theta^{-1}$ the theoretical solidity of the time reversible case.

Let $\underline{\underline{e}} D=\operatorname{diag}\left(e_{n}\right)$. Recall that $\sqrt{\underline{x}^{T} \underline{\underline{U}} \underline{x}}$ is a vector norm when $\underline{\underline{U}}$ is positive definite. The scalar function

$$
d(t)=\sqrt{\left(\underline{\underline{p}}^{T}(t)-\underline{e}^{T}\right) \underline{\underline{e}} \bar{D}^{-1}\left(\underline{p}^{T}(t)-\underline{e}^{T}\right)}
$$

is then a vector norm and a distance to ergodicity.

It has been shown by D.G. Kendall [3] that the distance $d(t)$ is monotone decreasing for time reversible chains. It has also been shown by Keilson and Vasicek [2] that this distance decreases to zero for all ergodic chains. An independent proof will be given in this paper.

\section{The Structure of the Distance to Ergodicity}

The structure of $d(t)$ for all finite ergodic chains is examined more deeply here. This structure is used to establish the relaxation time $\theta^{-1}$ entirely in the real domain without any reference to complex eigenvalues until the end. We use the following notation:

\section{Definitions:}
2a) $\quad \underline{\underline{e}}_{D}=\operatorname{diag}\left(e_{n}\right)$
2b) $\quad \underline{\nu}^{T}(t)=\left(\underline{p}^{T}(t)-\underline{e}^{T}\right) \underline{\underline{e}} \bar{D}^{-1 / 2}$
2c) $\quad w(t)=\underline{\nu}^{T}(t) \underline{\nu}(t)$
2d) $\quad d(t)=\sqrt{w(t)}$
2e) $\quad \underline{\underline{Q}}^{R}=\underline{\underline{e}}^{-1} \underline{\underline{Q}}^{T} \underline{\underline{e}} D$
2f) $\quad \underline{\underline{Q}}^{\#}=\frac{1}{2}\left[\underline{\underline{Q}}+\underline{\underline{Q}}^{R}\right]$
2g) $\quad \underline{\underline{B}}=\underline{\underline{e}} \underline{\underline{D}}^{1 / 2} \underline{\underline{Q}} \underline{\underline{e}} \bar{D}^{-1 / 2}$
2h) $\quad \underline{\underline{C}}=\frac{1}{2}\left[\underline{\underline{B}}+\underline{\underline{B}}^{T}\right]=\underline{\underline{e}}^{1 / 2} \underline{\underline{Q}}^{\#} \underline{\underline{e}} \bar{D}^{-1 / 2}$

The superscript $R$ refers to the reverse chain. Note that $\underline{\underline{Q}}, \underline{\underline{Q}}^{R}$, and $\underline{Q}^{\#}$ all generate chains which have the same ergodic vector $\underline{e}^{T}$.

Theorem A: For any finite ergodic Markov chain $J(t)$ :

(a) $\underline{Q}^{\#}$ is the $Q$-matrix of an ergodic chain;

(b) $\quad \underline{\underline{C}}=\underline{\underline{e}}_{D}^{1 / 2} \underline{\underline{Q}}^{\#} \underline{\underline{e}} \bar{D}^{-1 / 2}$ is symmetric and negative-semidefinite with eigenvalues $\lambda_{1}=0, \lambda_{j}<0, j \neq 1$.

(c) The stationary chain generated by $\underline{\underline{Q}}^{\#}$ is time-reversible.

Proof: $\underline{\underline{Q}}$ and $\underline{\underline{Q}}^{R}=\underline{\underline{e}} \bar{D}^{-1} \underline{\underline{Q}}^{T} \underline{\underline{e}} D$ have the zero structure needed to be an ergodic $Q$ matrix as does $\underline{\underline{Q}}^{\#}=\frac{1}{2}\left[\underline{\underline{Q}}+\underline{\underline{Q}}^{R}\right]$. The matrices $\underline{\underline{Q}}, \underline{\underline{Q}}^{R}$ and $\underline{\underline{Q}}^{\#}$ all have row sum zero. Also $2 \underline{\underline{e}}_{D}^{1 / 2} \underline{\underline{Q}}^{\#} \underline{\underline{e}}{ }_{D}^{-1 / 2}=\underline{\underline{e}}_{D}^{1 / 2} \underline{\underline{Q}} \underline{\underline{e}}{ }^{-1 / 2}+\underline{\underline{e}}_{D}^{1 / 2} \underline{\underline{Q}}^{R} \underline{\underline{e}}{ }_{D}^{-1 / 2}=\underline{\underline{e}}_{D}^{1 / 2} \underline{\underline{Q}} e^{-1 / 2}+$ $\underline{\underline{e}}-1 / 2 \underline{Q}^{T} \underline{\underline{e}} D^{1 / 2}$ is symmetric. Hence $\underline{\underline{e}} D \underline{\underline{Q}}^{\#}=\underline{e}_{D}^{1 / 2}\left[\underline{\underline{e}}^{1 / 2} \underline{\underline{Q}}^{\#} \underline{\underline{e}}-1 / 2\right] \underline{\underline{e}} D^{1 / 2}$ is symmetric and $J^{\#}(t)$ governed by $\underline{Q}^{\#}$ is time-reversible (cf. Keilson [1]).

Theorem B: Let $\theta=\min _{\lambda_{j} \neq 0}\left\{\lambda_{j}[-\underline{\underline{C}}]\right\}=\min _{\lambda_{j} \neq 0}\left\{\lambda_{j}\left[-\underline{\underline{Q}}^{\#}\right]\right\} . \quad T_{R E L}^{\#}=$ $\theta^{-1}$ is then the relaxation time of the ergodic time reversible chain $J^{\#}(t)$. For any finite ergodic Markov chain, it has been shown in [4] that 


$$
\frac{d(t)}{d(0)}=\sqrt{\frac{w(t)}{w(0)}} \leq e^{-\theta t} .
$$

The rate $\theta$ will be called the global decay rate of $d(t)=\sqrt{w(t)}$. The proof is given here.

Proof: From the definitions, one has at once $\frac{d}{d t} \underline{\nu}^{T}(t)=\underline{\nu}^{T}(t) \underline{\underline{B}}$ and $\frac{d}{d t} \underline{\nu}(t)=$ $\underline{\underline{B}}^{T} \underline{\nu}(t)$. Hence $\frac{d}{d t} w(t)=\frac{d}{d t}\left[\underline{\nu}^{T}(t) \underline{\nu}(t)\right]=\underline{\nu}^{T}(t) \underline{B} \underline{\nu}(t)+\underline{\nu}^{T}(t) \underline{\underline{B}}^{T} \underline{\nu}(t)$. This implies

$$
\frac{d}{d t} w(t)=2\left[\underline{\nu}^{T}(t) \underline{\underline{C}} \underline{\nu}(t)\right]
$$

Since $\underline{x}^{T} \underline{\underline{C}} \underline{x}<0$, for all real $\underline{x} \neq \underline{0}, \frac{d}{d t} w(t) \leq 0$ and $w(t)$ decreases in $t$. The matrix $\underline{\underline{C}}$ has principal left eigenvector $\underline{e}_{C}^{T}=\left(e_{n}^{1 / 2}\right)$ corresponding to eigenvalue 0 and $\left.\underline{\nu}^{T}(t) \underline{e}_{C}=\left(\underline{p}^{T} t\right)-\underline{e}^{T}\right) \underline{1}=0$. Thus $\underline{\nu}^{T}(t)$ is orthogonal to the principal rank one eigenspace of $\underline{\underline{C}}$. When $\underline{p}^{T}(0) \neq \underline{e}^{T}, \underline{\nu}^{T}(t)$ moves in this space, and does not vanish. One has from the Rayleigh-Ritz principal

$$
\frac{d}{d t} \log w(t)=2 \frac{\underline{\nu}^{T}(t) \underline{\underline{C}} \underline{\nu}(t)}{\underline{\nu}^{T}(t) \underline{\nu}(t)} \leq-\min _{\lambda_{j}>0}\left\{\lambda_{j}[-\underline{\underline{C}}]\right\}=-2 \theta .
$$

If one integrates from 0 to $t$, Theorem $\mathrm{B}$ follows.

Convexity Lemma: The function $w(t)$ is convex and

$$
\frac{d^{2}}{d t^{2}} w(t)=4 \underline{\nu}^{T}(t) \underline{\underline{C}}^{2} \underline{\nu}(t) \geq 0
$$

Proof: From $\frac{d}{d t} w(t)=\frac{d}{d t}\left[\underline{\nu}^{T}(t) \underline{\nu}(t)\right]=2 \underline{\nu}^{T}(t) \underline{\underline{C}} \underline{\nu}(t)$ one has

$$
\frac{d^{2}}{d t^{2}} w(t)=\frac{d}{d t}\left[\underline{\nu}^{T}(t) \underline{C} \underline{\nu}(t)\right]=2 \underline{\nu}^{T}(t)\left[\underline{\underline{B}} \underline{\underline{C}}+\underline{\underline{C}} \underline{\underline{B}}^{T}\right] \underline{\nu}(t)
$$

But

$$
2\left[\underline{\underline{B}} \underline{\underline{C}}+\underline{\underline{C}} \underline{\underline{B}}^{T}\right]=\underline{\underline{B}}\left[\underline{\underline{B}}+\underline{\underline{B}}^{T}\right]+\left[\underline{\underline{B}}+\underline{\underline{B}}^{T}\right] \underline{\underline{B}}^{T}=\left(\underline{\underline{B}}+\underline{\underline{B}}^{T}\right)^{2}+\left(\underline{\underline{B}} \underline{\underline{B}}^{T}-\underline{\underline{B}}^{T} \underline{\underline{B}}\right)
$$

and $\left(\underline{\underline{B}} \underline{\underline{B}}^{T}-\underline{\underline{B}}^{T} \underline{\underline{B}}\right)$ is antisymmetric. The lemma then follows.

A stronger result is available.

Theorem C: For any finite ergodic Markov chain, with $d(0) \neq 0$,

(a) $\quad w(t)$ is convex and decreasing in $t$;

(b) $\log w(t)$ is convex in $t$, i.e. $\frac{w^{\prime}(t)}{w(t)}$ increases with $t$;

(c) $\frac{w^{\prime}(t)}{w(t)} \leq-2 \theta$. This equality is tight, i.e., an initial state vector can be found for which $\frac{w^{\prime}(t)}{w(t)}=-2 \theta$ for all $t$.

Proof: From the proof of Theorem B, $w^{\prime}(t)<0$. We must show that $[\log w(t)]^{\prime \prime}=\frac{w(t) w^{\prime \prime}(t)-\left[w^{\prime}(t)\right]^{2}}{w^{2}} \geq 0$. Calculation gives

$$
w(t) w^{\prime \prime}(t)-\left[w^{\prime}(t)\right]^{2}=4\left\{\left[\underline{\nu}^{T}(t) \underline{\nu}(t)\right]\left[\underline{\nu}^{T}(t) \underline{\underline{C}}^{2} \underline{\nu}(t)\right]-\left[\underline{\nu}^{T}(t) \underline{\underline{C}} \underline{\nu}(t)\right]^{2}\right\}
$$

where $\underline{\underline{C}}=\underline{\underline{e}} \underline{D}^{1 / 2} \underline{\underline{Q}}^{\#} \underline{\underline{e}} \bar{D}^{-1 / 2}$. Moreover since $\underline{\underline{C}}$ is symmetric, the Schwartz inequality 
gives

$$
\left|\underline{\nu}^{T}(t) \underline{\underline{C}} \underline{\nu}(t)\right|^{2} \leq\left|\underline{\nu}^{T}(t) \underline{\underline{C}}\right|^{2}\left[\underline{\nu}^{T}(t) \underline{\nu}(t)\right]=\left[\underline{\nu}^{T}(t) \underline{\underline{C}}^{2} \underline{\nu}(t)\right]\left[\underline{\nu}^{T}(t) \underline{\nu}(t)\right]
$$

and $w(t) w^{\prime \prime}(t)-\left[w^{\prime}(t)\right]^{2} \geq 0$. This proves log-convexity.

For $\underline{p}^{T}(0) \neq \underline{e}^{T}$, the Schwartz inequality is strict and the convexity of $\log \frac{w(t)}{w(0)}$ is strict unless $\underline{\nu}^{T}(t) \underline{C}=K \underline{\nu}^{T}$ for some constant $K$. This can only happen when $\underline{\nu}^{T}(t)$ is an eigenvector of $\underline{\underline{C}}$, i.e., when $\left(\underline{p}^{T}(t)-\underline{e}^{T}\right) \underline{\underline{e}} \bar{D}^{-1 / 2} \underline{\underline{C}}=\lambda_{j}[\underline{\underline{C}}]\left(\underline{p}^{T}(t)-\underline{e}^{T}\right) \underline{\underline{e}} \bar{D}^{-1 / 2}$.

We next show that the inequality (3) in Theorem B is tight in that, for any ergodic chain one can always find a $\underline{p}^{T}(0)$ for which $\frac{w(t)}{w(0)}=\exp [-2 \theta] t$. If $\frac{w^{\prime}(0)}{w(0)}=-2 \theta$, knowledge that $\frac{w^{\prime}(t)}{w(t)}$ is increasing and $\frac{w^{\prime}(t)}{w(t)} \leq-2 \theta$ implies that $\frac{w^{\prime}(t)}{w(t)}=-2 \theta$ for all $t$. Let $\underline{u}_{1}^{T}$ be any real orthonormal eigenvector of $\underline{\underline{C}}$ for the eigenvalue $-\theta$, and $\alpha$ be small and real. If $\underline{\nu}^{T}(0)=\left(\underline{p}^{T}(0)-\underline{e}^{T}\right) \underline{e} \bar{D}^{-1 / 2}=\alpha \underline{u}_{1}^{T}$, so that $\underline{\nu}^{T}(0) \underline{C}=-\alpha \theta \underline{u}_{1}^{T}$ then $\frac{w^{\prime}(0)}{w(0)}=2 \frac{\underline{\nu}^{T}(0) \underline{\underline{L}} \underline{\nu}(0)}{\underline{\nu}^{T}(0) \underline{\nu}(0)}=-2 \theta$ as needed. If one chooses $\underline{p}^{T}(0)=\underline{e}^{T}+\alpha \underline{u}_{1}^{T} \underline{e}_{D}^{1 / 2}$, one will have $\underline{p}^{T}(0)>\underline{0}^{T}$ for $\alpha$ sufficiently small. Moreover one will also have $\underline{p}^{T}(0) \underline{1}=1$. For

$$
\left(\underline{p}^{T}(0)-\underline{e}^{T}\right) \underline{1}=\alpha \underline{u}_{1}^{T} \underline{e}_{D}^{1 / 2} \underline{1} \text { and }-\theta \underline{u}_{1}^{T} \underline{e}_{D}^{1 / 2} \underline{1}=\underline{u}_{1}^{T} \underline{C} \underline{\underline{e}} \underline{e}_{D}^{1 / 2} \underline{1}=\underline{u}_{1}^{T} \underline{e}_{D}^{1 / 2} \underline{\underline{Q}}^{\#} \underline{1}=0
$$

Time-reversible case: When $J(t)$ governed by $Q$ is time reversible, a special case

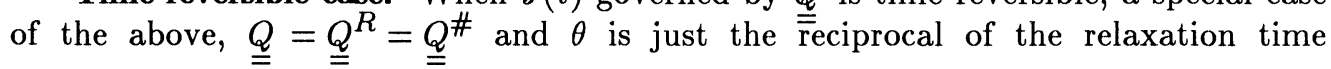
described in [1]. For this time reversible case, for any $\lambda_{j}$ and real eigenvector $\underline{u}_{j}^{T}$ of $\underline{\underline{Q}}$, one can find initial vectors $\underline{p}^{T}(0)$ for which $w(t)$ will have purely exponential decay at rate $2\left|\lambda_{j}\right|$ faster than $2 \theta$. The global decay rate is still $\theta$.

\section{The Covariance Function}

Let $J(t)$ be any finite ergodic Markov chain which is stationary. Let $f(j)$ be any real function of state $j$. The covariance function is $R_{f}(\tau)=\operatorname{cov}[f(J(t), f(J(t+\tau)]$ and (cf. [1]) $R_{f}(\tau)=\underline{f}^{T} \underline{\underline{e}}_{D}\left[\underline{\underline{p}}(\tau)-\underline{l} \underline{e}^{T}\right] \underline{f}$ for $\tau>0$.

The correlation function is $\rho_{f}(\tau)=\frac{R_{f}(|\tau|)}{R_{f}(0)}$.

Theorem D: For any finite ergodic Markov chain $J(t)$ which is stationary, the correlation function satisfies

$$
\left|\rho_{f}(\tau)\right| \leq \exp [-\theta|\tau|]
$$

and the inequality is tight.

Proof: Without loss of generality, we may assume that $f_{n}>0$ since a positive constant may always be added to $f_{n}$ without altering the covariance. Let $\underline{\underline{p}}(\tau)=$ 
$\left[p_{m n}(\tau)\right]$ be the transition probability matrix of $J(t)$. For $\underline{\nu}^{T}(\tau)=$ $\frac{\underline{f}^{T} \underline{\underline{e}} D}{\underline{f}^{T} \underline{\underline{e}} D \underline{1}}\left[\underline{\underline{p}}(\tau)-\underline{1} \underline{e}^{T}\right] \underline{\underline{e}} \underline{D}^{-1 / 2}$, algebra gives

$$
\begin{gathered}
R_{f}(\tau)=\underline{f}^{T} \underline{\underline{e}} D\left[\underline{\underline{p}}(\tau)-\underline{1} \underline{e}^{T} \underline{\underline{e}} \underline{D}^{-1 / 2} \underline{\underline{e}} \bar{D}^{-1 / 2}\left[\underline{\underline{e}} D \underline{f}-\left(\underline{f}^{T} \underline{\underline{e}} D \underline{1}\right) \underline{e}\right]\right. \\
=\left(\underline{f}^{T} \underline{\underline{e}} D \underline{1}\right)^{2}\left[\underline{\nu}^{T}(\tau) \underline{\nu}(0)\right] .
\end{gathered}
$$

From the Schwartz inequality,

$$
\rho_{f}^{2}(\tau)=\frac{R_{f}^{2}(\tau)}{R_{f}^{2}(0)}=\frac{\left[\underline{\nu}^{T}(\tau) \underline{\nu}(0)\right]^{2}}{\left[\underline{\nu}^{T}(0) \underline{\nu}(0)\right]^{2}} \leq \frac{|\underline{\nu}(\tau)|^{2}}{|\underline{\nu}(0)|^{2}}=\frac{w(\tau)}{w(0)} \leq \exp [-2 \theta \tau] .
$$

The tightness of the inequality follows from the tightness in Theorem C. This proves the theorem.

\section{The Relaxation Time}

In [1] the relaxation time was defined by $T_{R E L}=\sup _{f} \int_{0}^{\infty} \rho_{f}(\tau) d \tau$. This was motivated by the similarity of $\rho_{f}(\tau)$ to a survival function. One then has at once from Theorem $\mathrm{D}, T_{R E L}=\theta^{-1}$.

One must finally relate the decay rate $\theta$ to the eigenvalues of $\underline{\underline{Q}}$. Suppose that there are eigenvalues of $\underline{\underline{Q}}$ with negative real part $-\zeta$ and that other eigenvalues have a more negative real part. Consider $w(t)=\underline{\nu}^{T}(t) \underline{\nu}(t)=\left(\underline{p}^{T}(t)-\underline{e}^{T}\right) \underline{\underline{e}}{ }^{-1}(\underline{p}(t)-$ $\underline{e}) . \quad e^{2 \zeta t} w(t)=\left[e^{\zeta t}\left(\underline{p}^{T}(t)-\underline{e}^{T}\right)\right] \underline{\underline{e}}-{ }^{-1}\left[e^{\zeta t}(\underline{p}(t)-\underline{e})\right]=\left[e^{\zeta t} d(t)\right]^{2}$ and that this is logconvex in $t$, all $\zeta$. For $\underline{p}^{T}(t)-\underline{e}^{T} \neq 0$, $\limsup _{t \rightarrow \infty} e^{2 \zeta t} w(t)=0$, when $\zeta<-\theta^{*}$ and $\limsup _{t \rightarrow \infty} e^{2 \zeta t} w(t)=\infty$, when $\zeta>=\theta^{*}$. From the tightness in Theorem $C$, we must then identify $\theta^{*}$ with $\theta$.

A calculation has been carried out using the symbolic and numerical power of Maple for the chain $J(t)$ starting in any state and generator

$$
\underline{Q}=\left[\begin{array}{ccccc}
-1 & 1 & 0 & 0 & 0 \\
0 & -1 & 1 & 0 & 0 \\
0 & 0 & -1 & 1 & 0 \\
0 & 0 & 0 & -1 & 1 \\
1 & 0 & 0 & 0 & -1
\end{array}\right]
$$

The graph given by Maple is found to be log-convex as predicted. The symbolic expression for $w(t)$ has the asymptotic decay rate predicted. 


\section{Acknowledgement}

The author wishes to thank P. Gundelpudi and A. Roy for their interest and help.

\section{Appendix}

Lemma: If $\lambda_{j}[Q]$ is the eigenvalue of an ergodic finite Markov chain in continuous

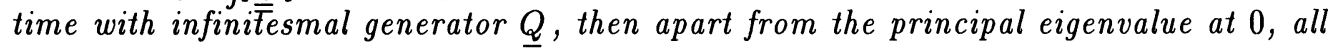
eigenvalues of $\underline{Q}$ have a strictly $n \overline{\bar{e}}$ gative real part.

Proof: We may use uniformization [1] to write $\underline{\underline{Q}}=-\nu\left[\underline{\underline{I}}=\underline{\underline{a}}_{\nu}\right]$ where $\nu$ is any positive rate exceeding the largest exit rate from a state and $\underline{a}_{\nu}$ is a stochastic ergodic (irreducible and aperiodic) matrix. Then $\lambda_{j}[\underline{\underline{Q}}]=-\nu\left(1-\lambda_{j}\left[\underline{\underline{a}}_{\nu}\right]\right)$ and $\left|\lambda_{j}\left[\underline{\underline{a}}_{\nu}\right]\right|<1$ for other than $\lambda_{1}\left[\underline{\underline{a}}_{\nu}\right]=1$. The lemma then follows.

Remark: One can have a stochastic matrix which is ergodic and has purely imaginary eigenvalues.

An example with eigenvalues $1,-\frac{1}{2}, \frac{1}{2} i,-\frac{1}{2} i$ is

$$
\left[\begin{array}{cccc}
\frac{1}{8} & \frac{5}{8} & \frac{1}{8} & \frac{1}{8} \\
\frac{1}{8} & \frac{1}{8} & \frac{5}{8} & \frac{1}{8} \\
\frac{1}{8} & \frac{1}{8} & \frac{1}{8} & \frac{5}{8} \\
\frac{5}{8} & \frac{1}{8} & \frac{1}{8} & \frac{1}{8}
\end{array}\right] .
$$

\section{References}

[1] Keilson, J., Markov Chain Models - Rarity and Exponentiality, Springer-Verlag, Applied Mathematical Sciences Series 281979.

[2] Keilson, J. and Vasicek, O.A., Monotone measures of ergodicity for Markov chains, Special Issue for Ryszard Syski, J. of Appl. Math. and Stoch. Anal., 11:3 (1998), 281-286.

[3] Kendall, D.G., Unitary Dilations of Markov Transition Operators and the Corresponding Integral Representations for Transition Probability Matrices, (ed. by U. Grenander), Probability and Statistics, Almqvist and Wiksell, Stockholm 1959.

[4] Roy, Abhijit, Transient Analysis of Queueing Systems, Ph.D. Thesis, The University of Rochester (1996). This is available from UMI, Ann Arbor, MI. 


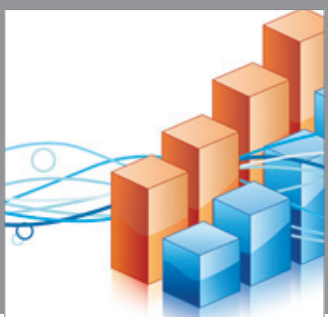

Advances in

Operations Research

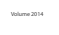

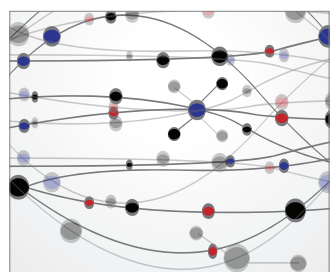

\section{The Scientific} World Journal
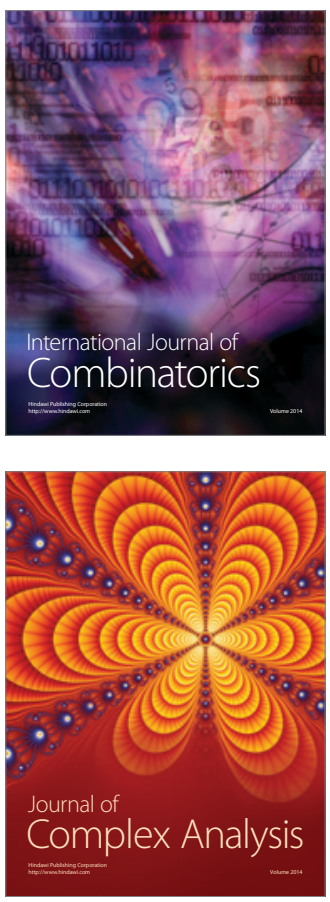

International Journal of

Mathematics and

Mathematical

Sciences
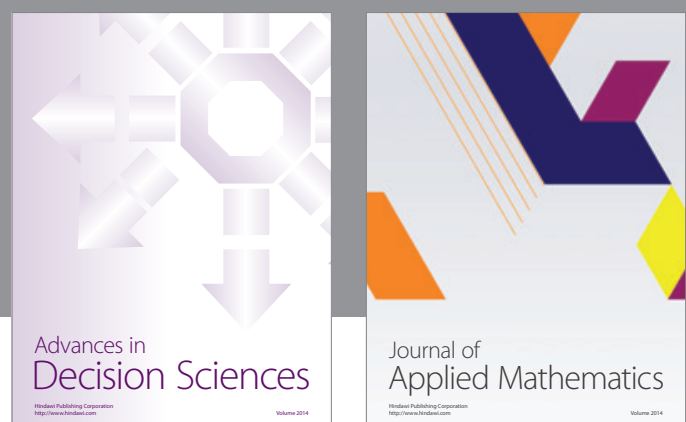

Journal of

Applied Mathematics
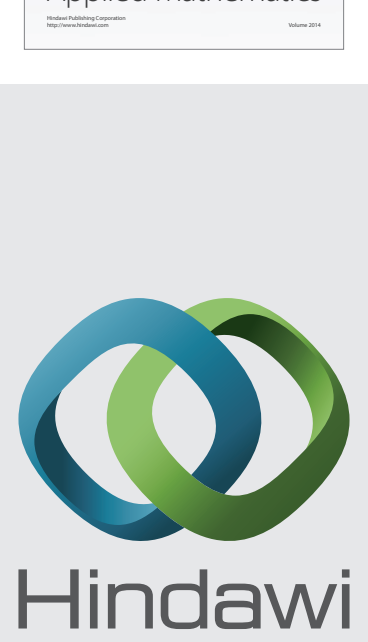

Submit your manuscripts at http://www.hindawi.com
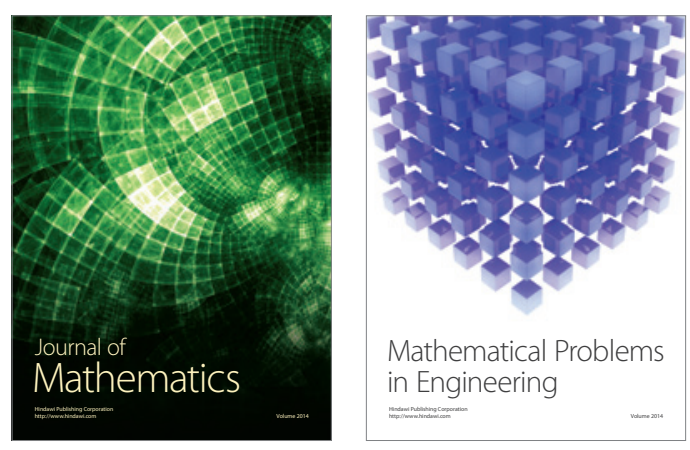

Mathematical Problems in Engineering
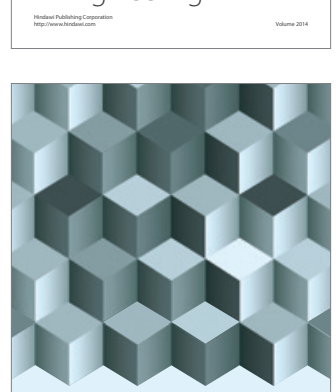

Journal of

Function Spaces
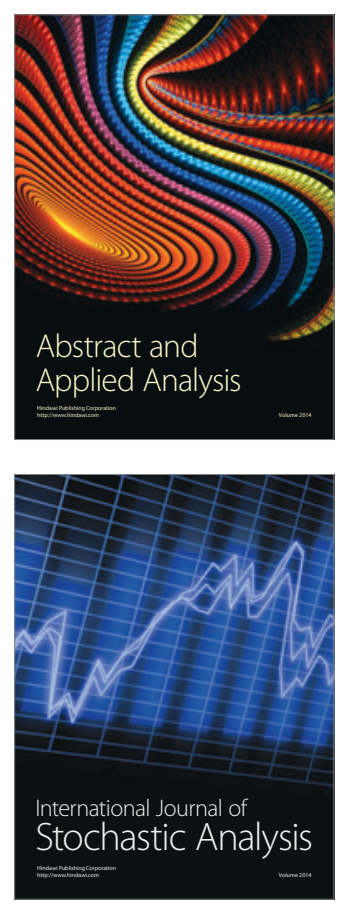

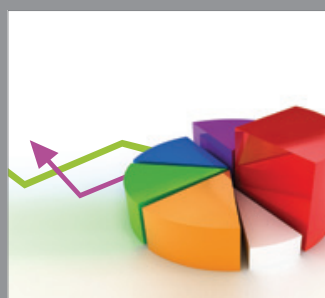

ournal of

Probability and Statistics

Promensencen
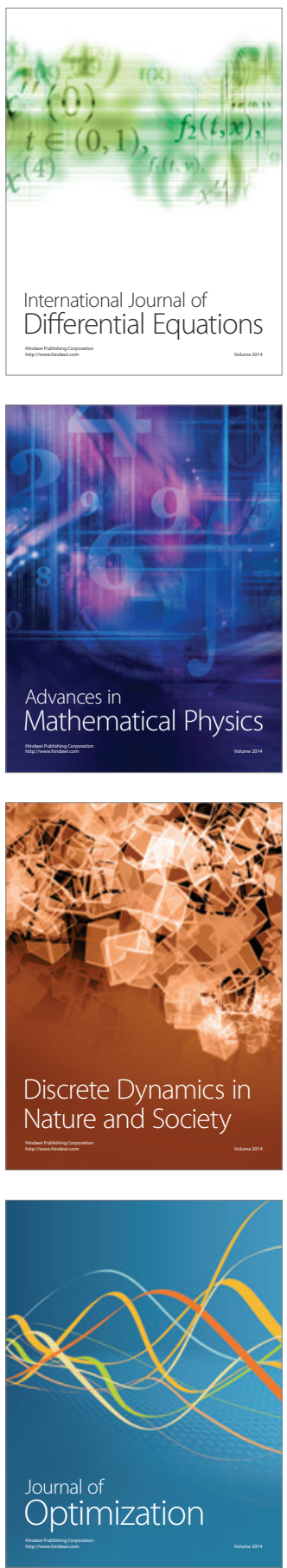